\title{
Dietary Sodium Intake and Arterial Blood Pressure
}

Francis Dumler MD

Division of Nephrology

William Beaumont Hospital

Royal Oak, MI USA

Address correspondence to: Francis Dumler MD

Division of Nephrology

William Beaumont Hospital

Royal Oak, MI 48009

USA

Telephone: 248-551-1055

Facsimile: 248-551-3689

Email 1: fdumler@beaumont.edu

Email 2: fdumler@yahoo.com 


\begin{abstract}
OBJECTIVE: to summarize major recent studies in the field.

INTERSALT: sodium intake correlates with the rise in blood pressure with age, but not with the prevalence of hypertension. The population study identified a minimal impact of sodium intake on blood pressure $(0.9 \mathrm{~mm} \mathrm{Hg} / 10 \mathrm{mmol}$ difference in salt intake).
\end{abstract}

DASH: this diet induced significant reductions in blood pressure when compared to the control diet. Further decreases were observed with DASH and a $50 \mathrm{mmol} /$ day sodium intake.

VANGUARD: blood pressure was inversely related to urinary potassium, calcium and magnesium but not to sodium excretion.

TONE: cardiovascular events were highest in the usual care group (83\%) and lowest in the sodium reduction plus weight loss group (56\%).

META-ANALYSIS: a systematic review of eleven long term controlled randomized trials reported a small decrease $(1.1 \mathrm{~mm} \mathrm{Hg})$ in median systolic but not diastolic blood pressure with a reduced dietary sodium intake.

CONCLUSIONS: 1) Sodium restriction in hypertensive patients reduces blood pressure. 2) The long term impact of reduced salt intake on blood pressure, mortality, and morbidity remains to be defined. 
Introduction

Systemic arterial pressure is regulated by complex interactive systems, including the circulatory volume, vascular resistance vessels, central nervous system, kidneys, and endocrine components. The relationship between salt consumption and blood pressure continues to generate considerable discussion and debate. Kempner used a very low-salt rice diet ( $0.6 \mathrm{~g}$ sodium, $5 \mathrm{~g}$ fat, $20 \mathrm{~g}$ protein per day) to reduce blood pressure in patients with malignant hypertension and renal failure (1). Guyton showed that a rise in blood pressure caused a pressure sodium diuresis that reduced vascular volume and lowered blood pressure. He concluded that renal compensation is reduced in essential hypertension (2). An anatomical counterpart to this reduced compensation to dietary salt intake is the decreased nephron number reported in patients with essential hypertension (3). Dahl correlated the prevalence of HTN in five geographically distinct populations with their average daily salt intake and published his results in a monograph (4). He proposed that the prevalence of hypertension rises linearly with salt consumption between 4 and 26 grams per day. Many population studies have followed, but results have often been conflicting.

\section{NHLBI INTERSALT STUDY}

This is a multicenter study of 10,079 patients in 52 centers around the world. Sodium excretion ranged from 0.12 to $242 \mathrm{mmol} / 24$ hours (5). Subjects in the four remote areas (Yanomamo and Xingu in Brazil, Papua-New Guinea, and Kenya) had the lowest sodium excretion and were all within the lowest quintile (6). Systolic blood pressure showed a minimal or no increase by age 55. Diastolic blood pressure had a discrete increase only in the Kenya cohort. The prevalence of hypertension was very low (Yanomamo 0\%, Xingu 1\%, Papua New Guinea 1\%, and Kenya 3\%) (7). Across the other 48 centers, sodium intake was significantly related to a rise in blood pressure with age. No correlation was found between sodium and median blood pressure or the prevalence of hypertension. More important, no correlation was found between sodium excretion and blood pressure in the cross sectional population study unless the four outliers (remote population subgroup) were included in the analysis. The relationship between blood pressure and age was stronger in populations with high rather than low salt intake. However, the increase was rather small $(0.9 \mathrm{~mm} \mathrm{Hg}$ for each $10 \mathrm{mmol}$ increase in salt intake).

Population studies, such as INTERSALT, have built-in limitations. There are significant ecological and cultural differences among population centers. Spot blood pressure and sodium excretion values are extremely variable between centers and within individuals. In order to adjust for multiple confounders and to correct for dilutional regression, a reanalysis of the entire data was done in 1996 (8). These authors reported a strong association between urinary sodium and systolic pressure in both individuals and population centers. Others felt that such 
manipulation significantly undermined the credibility of the INTERSALT study $(9,10)$. An alternative explanation is that there is a threshold for the effect of sodium intake on blood pressure at around $100 \mathrm{mmol}$ per day (11).

Differences in response to salt intake may also be related to time exposure and varying salt sensitivity (12). A meta-analysis study of mostly crossover design noted that trials lasting up to four weeks reduced blood pressure less than those carried out for five weeks or longer (13). A $50 \mathrm{mmol}$ reduction in daily sodium intake lowered systolic blood pressure by $5-7 \mathrm{~mm} \mathrm{Hg}$ and diastolic values by $1.5-2.5 \mathrm{~mm} \mathrm{Hg}$. Based on these results Law et al. argued for a universal dietary salt reduction. A second large meta-analysis reported somewhat different conclusions (14). In this study a reduced dietary sodium intake was associated with a $3.9 \mathrm{~mm} \mathrm{Hg}$ decrease in systolic and a $1.9 \mathrm{~mm} \mathrm{Hg}$ reduction in diastolic blood pressure in hypertensive subjects. In normotensive individuals, lowering dietary sodium intake decreased systolic and diastolic blood pressure only by $1.2 \mathrm{~mm} \mathrm{Hg}$ and $0.26 \mathrm{~mm} \mathrm{Hg}$ respectively. The authors concluded there was no supportive evidence for a general recommendation to reduce sodium intake. They did suggest a reduced sodium intake as a supplementary treatment of hypertension.

\section{DIETARY APPROACHES TO STOP HYPERTENSION TRIAL (DASH)}

This is the most comprehensive study of diet intervention to date $(15,16)$. In DASH I, adults with blood pressure $<160 / 80-95 \mathrm{~mm} \mathrm{Hg}$ were randomized to an average American diet (control), a diet rich in fruits and vegetables, or a combination diet (DASH) rich in fruits and vegetables, with a low content of saturated and total fats. Sodium intake was maintained constant at 3 grams per day. The DASH diet reduced systolic and diastolic blood pressure by 5.5 and $3.0 \mathrm{~mm} \mathrm{Hg}$ respectively. Hypertensive patients had greater falls in blood pressure when compared to normotensive subjects (11.4 and 5.5 versus 3.5 and $2.1 \mathrm{~mm} \mathrm{Hg}$ for systolic and diastolic blood pressure respectively). DASH II addressed the added impact of a low sodium intake. Blood pressure progressively decreased in both the control and DASH groups as dietary sodium was lowered from 150 to $50 \mathrm{mmol} /$ day. The changes were more pronounced with the DASH diet.

Subset analysis of DASH-I identified greater systolic blood pressure reductions in hypertensive than in normotensive (11.4 and $3.4 \mathrm{~mm} \mathrm{Hg}$ respectively) subjects (17). African Americans had greater decreases than whites (6.8 and $3.0 \mathrm{~mm} \mathrm{Hg}$ respectively). Subjects older than age 45 had larger reductions in blood pressure $(7.0 / 3.8 \mathrm{~mm} \mathrm{Hg})$ when compared to younger ones $(3.7 / 1.5 \mathrm{~mm} \mathrm{Hg})(18)$. This suggests that increased sodium sensitivity may be limited to the older non hypertensive population.

Subjects assigned to the DASH diet showed significantly greater decreases in homocysteine levels than those on the control diet. This change was significantly and inversely related to serum folate levels but not vitamin B6 or B12 (19). Relative to the control diet, the DASH cohort significantly lowered their total $(-13.7 \mathrm{mg} / \mathrm{dL}), \mathrm{LDH}(-10.7 \mathrm{mg} / \mathrm{dL})$ and HDL $(-3.7$ $\mathrm{mg} / \mathrm{dL}$ ) cholesterol (20). 
Results from clinical trials do not always translate into clinical practice because of different population characteristics and levels of adherence to the treatment protocols. The PREMIER study evaluated 810 individuals randomized to three groups (advice only, established intervention, and established + DASH intervention) (21). The prevalence of hypertension decreased similarly in both intervention groups. This was attributed to a lower adherence rate to the DASH guidelines or to the sub-additive nature of intervention effects on blood pressure. Folsom et al. have published a provocative study using individuals enrolled in the lowa Women's Health Study (IWHS) (22). Their primary aim was to assess if a greater concordance to DASH guidelines was associated with a reduced incidence of hypertension and cardiovascular mortality in initially non-hypertensive older women. In univariate analysis a trend was found for a decreasing incidence of hypertension and cardiovascular death as concordance with DASH guidelines increased. However, after adjusting for other risk factors this relationship was lost. The authors concluded that a very high concordance, as achieved in the DASH trials, may be needed for full benefit. Alternatively DASH may not be as effective in older Caucasian and initially normotensive women.

\section{VANGUARD STUDY}

The VANGUARD trial was designed to evaluate the impact of a comprehensive nutritional program on blood pressure and to better define physiologic mechanisms underlying the antihypertensive effects of such therapies (23). The study diet conformed to the RDA standards specified by the National Academy of Sciences, American Diabetes Association, American Heart Association, and others to provide optimal levels of macro- and micronutrients recommended for overall cardiovascular risk reduction (24). Subjects were assigned to a prepared (CCNW) or a selfselected (SSD) meal plan.

Reductions in blood pressure where higher with CCNW than SSD meals for systolic ($6.2 \pm 0.1$ vs. $-4.2 \pm 0.8 \mathrm{~mm} \mathrm{Hg}$ ) and diastolic $(-6.2 \pm 0.1$ vs. $-4.2 \pm 0.8 \mathrm{~mm} \mathrm{H})$ blood pressures. Neither basal blood pressure nor its change was related to absolute or diet-induced changes in urinary sodium excretion. However, diastolic blood pressure was significantly and inversely related to basal urinary potassium excretion $(P<0.001)$. In addition, the greater the rise in urinary and serum potassium, the greater the fall in systolic and diastolic blood pressure $(P<0.002)$. Basal systolic blood pressure was also directly related to urinary calcium excretion $(P<0.0018)$, specially in hyperlipidemic subjects. Urinary calcium, when considered in relation to urinary sodium $\left(U_{\mathrm{Ca}} / \mathrm{U}_{\mathrm{Na}}\right)$, predicted diet-induced changes in both systolic $(\mathrm{P}<0.001)$ and diastolic $(\mathrm{P}<$ $0.021)$ blood pressure. Diet induced changes in blood pressure were also related to changes in magnesium excretion $(P<0.05)$.

The authors emphasize the importance of other minerals, namely calcium, magnesium and phosphorus in the pathogenesis of hypertension. This is in agreement with other studies which link the blood pressure response to calcium with sodium excretion. They postulate a lowered $\mathrm{Ca} / \mathrm{Na}$ ratio (deficient calcium or excess salt) as a predisposing to higher blood 
pressure in salt-sensitive persons, which is reversed by restricting sodium or increasing calcium. They use these arguments to support the ionic hypothesis of cardiovascular and metabolic disease. The appeal of this concept is that it fits well the close relationship between hypertension, obesity, insulin resistance, and type- 2 diabetes.

\section{TRIAL OF NONPHARMACOLOGIC INTERVENTIONS IN THE ELDERLY (TONE)}

The TONE study evaluated the efficacy of sodium restriction and weight reduction on cardiovascular disease endpoints at 30 months in older patients with hypertension (25). The rate of cardiovascular events was $83 \%$ in the usual care group, $60 \%$ in the low sodium intake cohort, $62 \%$ in the weight reduction arm, and $56 \%$ in the combined sodium and weight reduction group. The authors concluded that low sodium intake and weight loss are effective and safe therapies capable of reducing cardiovascular events in elderly hypertensive patients.

\section{IMPACT OF SODIUM RESTRICTION ON BLOOD PRESSURE - SYSTEMATIC REVIEW}

As noted earlier, large systematic reviews on the effect of sodium restriction on blood pressure have reported conflicting results $(13,14)$. A recent meta-analysis evaluated eleven long-term randomized controlled trials with 3,491 participants (26). It was not possible to assess the impact on quality of life, mortality, or cardiovascular events as the number of occurrences were minimal. A mean decrease of $1.1 \mathrm{~mm} \mathrm{Hg}$ in systolic blood pressure was noted $(P<0.002)$. No effect was observed in diastolic blood pressure. The authors concluded that intense interventions (likely unsuited to primary care or population prevention programs) produce uncertain effects on mortality and cardiovascular events while attaining only small reductions in blood pressure.

\section{CONCLUSIONS}

From a mechanistic perspective the relationship between dietary sodium intake and blood pressure can be described as: a) dietary salt intake is a key factor in the development of hypertension; b) dietary salt intake is a factor only when considered in relation to calcium, magnesium, and potassium. Interventional trials are unlikely to solve this query as nutritional therapies for prevention and/or control of hypertension operate via multiple complex and interactive pathways that can't be reduced to a few specifics.

There is general consensus on the following: a) restricting sodium intake in patients with hypertension reduces blood pressure; b) a lower dietary salt intake may help patients stop blood pressure medications while maintaining good control; c) the long term effects of reduced salt intake on blood pressure, mortality, and morbidity in a population with and without hypertension is yet undefined. 
Confounding issues include: a) few deaths and cardiovascular events have been reported in salt reduction trials; b) systematic reviews show only a discrete effect on blood pressure (1.1 $\mathrm{mm} \mathrm{Hg}$ systolic and $0.6 \mathrm{~mm} \mathrm{Hg}$ diastolic with sodium reductions of 25\%); c) interventions used in clinical trials are highly intensive and not suited to primary care or prevention programs; d) data on the effects of dietary sodium reduction per se on overall health is sparse. 


\section{REFERENCES}

1. Kempner W: Treatment of hypertensive vascular disease with rice. Am J Med 4:545-577, 1948

2. Guyton AC: Long-term arterial pressure control: an analysis from animal experiments and computer and graphic models. Am J Physiol 259:R865-r877, 1990

3. Keller G, Zimmer G, Ritz E, et al: Nephron number in patients with primary hypertension. N Engl J Med 348:101-108, 2003

4. Dahl LK: Possible role of salt intake in the development of essential hypertension. In: Bock KD, Cattier PT, eds. Essential hypertension. Berlin: Springer-Vela, 53, 1960

5. Intersalt Cooperative Research Group: Intersalt: an international study of electrolyte excretion and blood pressure. Results for 24-hour urinary sodium and potassium excretion. BMJ 297:319-328, 1988

6. Rose G, Day S: The population mean predicts the number of deviant individuals. BMJ 301:1031-1034, 1990

7. Carvalho JJ, Baruzzi RG, Howard PF, et al: Blood pressure in four remote populations in the INTERSALT Study. Hypertension14:238-246, 1989

8. Elliott $P$, Stamler J, Nichols R, et al: Intersalt revisited: further analyses of 24 hour sodium excretion. BMJ 312:1249-1253, 1996

9. O'Shaughnessy KM, Karat FE: Salt handling and hypertension. J Clin Invest 113:1075-1081, 2004

10. McCarron DA: The dietary guideline for sodium: should we shake it up? Yes. Am J Clin Nutr 71:1013-1019, 2000

11. Kaplan NM: The dietary guideline for sodium: should we shake it up? No. Am J Clin Nutr71:1020-1026, 2000

12. Luft FC, Weinberger $\mathrm{MH}$ : Heterogeneous responses to changes in dietary salt intake: the salt-sensitivity paradigm. Am. J. Clin. Nutr. 65(Suppl. 2):612S-617S, 1997

13. Law MR, Frost CD, Wald NJ, et al: III - Analysis of data from trials of salt reduction. BMJ 302:819-824, 1991

14. Graudal NA, Galloe AM, Garred P: Effects of sodium restriction on blood pressure, renin, aldosterone, catecholamines, cholesterols, and triglyceride. A Meta-analysis. JAMA 279:1383-1391, 1998

15. Apple $L$, Moore TJ, Obarzank E, et al: A clinical trial of the effects of dietary patterns on blood pressure. N Engl J Med 336:1117-1124, 1997

16. Sacks FM, Svetkey LP, Vollmer WM, et al: Effects on blood pressure of reduced dietary sodium and the dietary approaches to stop hypertension (DASH) diet. N Engl J Med 344:3-10, 2001

17. Svetkey LP, Simons-Morton D, Vollmer WM, et al: Effect of dietary patterns on blood pressure. Subgroup analysis of the Dietary Approaches to Stop Hypertension (DASH) randomized clinical trial. Arch Intern Med159:285-293, 1999

18. Vollmer WM, Sacks FM, Ard J, et al: Effects of diet and sodium intake on blood pressure: subgroup analysis of the DASH-sodium trial. Ann Intern Med 135:1019-1028, 2001 
19. Apple $\sqcup$, Miller ER, Jee SH, et al: Effect of dietary patterns on serum homocysteine: results of a randomized controlled feeding study. Circulation 102:852-857, 2000

20. Obarzanek E, Sack FM, Vollmer WM, et al: Effects on blood lipids of a blood pressurelowering diet: the Dietary Approaches to Stop Hypertension (DASH) trial. Am J Clin Nutr 74:80-89, 2001

21. PREMIER Collaborative Research Group: Effects of comprehensive lifestyle modification on blood pressure control: Main results of the PREMIER clinical trial. JAMA 289:2083-2093, 2003

22. Folsom AR, Parker ED, Harnack LJ: Degree of concordance with DASH diet guidelines and incidence of hypertension and fatal cardiovascular disease. AM J Hypertens 20:225-232, 2007

23. Resnick LM, Oparil S, Chait A, et al: Factors affecting blood pressure responses to diet: the Vanguard study. Am J Hypertens. 2000;13:956-965, 2000

24. McCarron DA, Oparil S, Chait A, et al: Nutritional management of cardiovascular risk factors. Arch Intern Med 157:169-177, 1997

25. Whelton PK, Appel LJ, Espeland MA, et al: Sodium Reduction and Weight Loss in the Treatment of Hypertension in Older Persons: A Randomized Controlled Trial of Nonpharmacologic Interventions in the Elderly (TONE). JAMA 279:839-846, 1998

26. Hooper L, Bartlett C, Smith GD, et al: Systematic review of long term effects of advice to reduce dietary salt in adults. BMJ 325:628-636, 2002 